\title{
Study protocol for the development of a European measure of best practice for people with long term mental health problems in institutional care (DEMoBinc)
} Helen Killaspy*1, Michael King1, Christine Wright ${ }^{2}$, Sarah White ${ }^{2}$, Paul McCrone ${ }^{3}$, Thomas Kallert ${ }^{4}$, Jorge Cervilla ${ }^{5}$, Jiri Raboch ${ }^{6}$, Georgi Onchev7, Roberto Mezzina ${ }^{8}$, Durk Wiersma9 ${ }^{9}$, Andrzej Kiejna ${ }^{10}$, Dimitris Ploumpidis ${ }^{11}$ and Jose Miguel Caldas de Almeida ${ }^{12}$

\begin{abstract}
Address: ${ }^{1}$ Department of Mental Health Sciences, University College London, 2nd Floor, Royal Free Hospital, London, NW3 2PF, UK, ${ }^{2}$ Department of Mental Health, St George's University London, Cranmer Terrace, London, SW17 ORE, UK, ${ }^{3}$ King's College London, Health Service and Population Research Department, Institute of Psychiatry, De Crespigny Park, London, SE5, 4 Department of Psychiatry and Psychotherapy, University Hospital, Technische Universitaet Dresden, Fetscherstrasse 74, 01307 Dresden, Germany, ${ }^{5}$ Department of Psychiatry, University of Granada, Cuesta del Hospicio s/n, Granada, 18071, Spain, ${ }^{6}$ Psychiatric Department of the 1st Faculty of Medicine, Charles University, Ovocný trh 5, Praha 1, 116 36, Czech Republic, ${ }^{7}$ Department of Psychiatry, Medical University Sofia, St. Georgi Sofiisky str. 1, Sofia 1431, Bulgaria,

${ }^{8}$ Dipartimento di Salute Mentale, Via Sai, 1-3, Trieste, 34127, Italy, ${ }^{9}$ University Medical Centre, Hanzeplein 1, Groningen, 9700 RB, Netherlands, ${ }^{10}$ Department of Psychiatry, Wroclaw Medical University, Pasteura 1, Wroclaw, 50-367, Poland, ${ }^{11}$ University Mental Health Research Institute, Soranou Tou Efessiou 2, Athens 11527, Greece and ${ }^{12}$ Caldas de Almeida, Faculdade de Ciências Médicas, Universidade Nova de Lisboa, Campo dos Mártires da Pátria 130, 1169-056 Lisbon, Portugal
\end{abstract}

Email: Helen Killaspy* - h.killaspy@medsch.ucl.ac.uk; Michael King - m.king@medsch.ucl.ac.uk; Christine Wright - cwright@sgul.ac.uk; Sarah White - swhite@sgul.ac.uk; Paul McCrone - p.mccrone@iop.kcl.ac.uk; Thomas Kallert - thomas.kallert@tu-dresden.de; Jorge Cervilla - jacb@ugr.es; Jiri Raboch - raboch@nbox.cesnet.cz; Georgi Onchev - georgeonchev@hotmail.com; Roberto Mezzina - roberto.mezzina@ass1.sanita.fvg.it; Durk Wiersma - Durk.Wiersma@med.umcg.nl;

Andrzej Kiejna - akiejna@psych.am.wroc.pl; Dimitris Ploumpidis - diploump@med.uoa.gr; Jose Miguel Caldas de Almeida - caldasjm@fem.uml.pt

* Corresponding author

Published: 13 June 2009

BMC Psychiatry 2009, 9:36 doi:10.1186/147/-244X-9-36

This article is available from: http://www.biomedcentral.com/I47I-244X/9/36

(C) 2009 Killaspy et al; licensee BioMed Central Ltd.

This is an Open Access article distributed under the terms of the Creative Commons Attribution License (http://creativecommons.org/licenses/by/2.0), which permits unrestricted use, distribution, and reproduction in any medium, provided the original work is properly cited.

\section{Abstract}

Background: This study aims to build a measure for assessing and reviewing the living conditions, care and human rights of people with longer term mental health problems in psychiatric and social care institutions. Protection of their human rights is imperative since impaired mental capacity secondary to mental illness can make them vulnerable to abuse and exploitation from others. They also constitute a major resource pressure for mental health services, social services, informal carers and society as a whole.

\section{Methods/Design:}

domains are identified by collating results from: i) a systematic review of the literature on institutional care for this service user group; ii) a review of the relevant care standards in each participating country; iii) Delphi exercises in partner countries with mental health professionals, service users, carers and advocates. Common domains and cross-cutting themes are agreed by the principal researchers and an international expert panel. Items are developed to assess these domains and incorporated into the toolkit which is designed to be administered through a face to 
face interview with the institution's manager. The toolkit is refined in response to inter-rater reliability testing, feedback from interviewers and interviewees regarding its utility, and feedback from key stakeholders in each country about its ability to deliver information that can be used within each country's established systems for quality assessment and review. Cross-validation of the toolkit ratings against service users' quality of life, autonomy and markers of recovery tests whether it can deliver a proxy-measure of the service users' experiences of care and the institution's promotion of their human rights and recovery. The ability of the toolkit to assess the "value for money" delivered by institutions is investigated by comparing toolkit ratings and service costs.

The study will deliver the first international tool for the assessment of the quality of institutional care for people with longer term mental health problems that is accurate, reliable, informative, useful and easy to use.

\section{Background}

The study began in March 2007 and is funded for three years by the European Commission. It involves ten countries and aims to develop a toolkit for assessing and reviewing the living conditions, care and human rights of people with mental health problems who require longer term care in a psychiatric or social care institution. No such international, standardised assessment tools currently exist. The majority of people living in these kinds of institution have a diagnosis of schizophrenia or schizoaffective disorder [1], major mental illnesses which have a chronic or fluctuating prognosis in up to $60 \%$ of cases [2]. By definition, these individuals are also likely to have additional characteristics which have complicated their recovery such that they require ongoing institutional care. These may include: treatment resistance (persistence of psychotic symptoms despite appropriate medication) which occurs in up to $30 \%$ [3]; cognitive impairment, specifically in the areas of executive function, verbal memory and pervasive negative symptoms such as apathy, amotivation and blunted affect [4-7]; pre-morbid learning disability and poor function [8]; drug and/or alcohol misuse and challenging behaviours [4,9]. Despite their high levels of need and, possibly because of the complexity of their problems, there is very little evidence for effective interventions available to guide practitioners. As well as the significant clinical challenges they pose for professionals, these individuals constitute a major resource pressure for mental health services, social services, informal carers and society as a whole since they require high levels of support and are usually unable to work.

Many social care institutions that provide for this group are within the independent sector. In England, there has recently been considerable concern about the variability of care provided and social dislocation for the individuals placed in these institutions in terms of distance from their family home and dislocation from local communities, particularly for those from black and ethnic minorities
[10-14]. This heterogeneity of service provision and quality is likely to be greater across countries.

Across Europe, countries are at different stages of deinstitutionalisation [15]. As such it is important that the toolkit covers themes that are both relevant and common to mental health services in different countries which are at different stages of development. Attention to the human rights of service users in these institutions is imperative since their mental health problems may impact on their capacity to make informed choices for themselves and to participate actively in their care. They are at risk of exploitation and abuse from others, including those who care for them. In addition, under resourcing of services can lead to practices which infringe civil liberties and deny basic human rights such as the right to privacy, choice and dignity. The European Commission's Green Paper "Improving the mental health of the population. Towards a strategy on mental health for the European Union" specifically highlights "the promotion of social inclusion of mentally ill or disabled people and protecting their fundamental rights and dignity". Quality of life and autonomy are key markers of whether clients' human rights are being promoted. Autonomy entails the freedom to choose from a range of options without persuasion or duress to influence that choice. However, the ability to choose can be affected by mental incapacity secondary to mental illness [16]. Quality of life is a global measure of an individual's well-being that can be affected by his or her human rights such as privacy, dignity, the absence of abuse and social inclusion [17]. Promotion of service users' autonomy should be a priority for these institutions since the negative effects of "institutionalisation" are well known [18].

\section{The Recovery framework}

We chose to use the concept of "Recovery" as an overarching framework in the development of the toolkit since it incorporates these issues and offers a model for services that is relevant across different countries. The Recovery 
approach [19] specifically advocates non-coercive relationships between professionals and service users that include negotiation of treatment plans and facilitation of patient autonomy. The "Recovery movement" originated in the 1990s in America from service users' narratives challenging the pessimistic prognoses of mental health professionals. It focuses on the individual's adaptation to the mental illness and optimism for the future with the acknowledgement that pre-morbid functioning may not be fully regained [20]. It is corroborated by recent long term outcome studies which have shown encouraging results for the majority of people diagnosed with schizophrenia [21], even those with high levels of needs [9]. Mental health services are increasingly encouraged to adopt a "Recovery style" in their practice [22,23]. Features of Recovery based practice include: collaborative working between staff and service users; therapeutic risk-taking such as negotiated "drug holidays"; and service user empowerment. Service user markers of Recovery have also been suggested [24] such as working, studying and participating in leisure activities in mainstream settings; good family relationships; living independently; having control of one's self-care, medication and money; having a social life; taking part in the local community; and satisfaction with life. Some of these are also markers of social inclusion.

\section{Methods and design}

Rationale for the method users' human rights and Recovery. This may mean that service user interviews will not be necessary in assessing institutions with the toolkit, which would maximise its practical application given the specific challenges that can arise in interviewing this service user group (secondary to their mental health problems). Having said this, assessors of institutions may, of course, wish to include interviews with service users. Our study design therefore aims to deliver a product (the toolkit) that allows flexibility in this to suit the specific setting.

\section{Setting and inclusion criteria}

erlands, Poland, Spain, Portugal and UK) selected to provide a range of different stages of deinstitutionalisation and a broad geographical and cultural spread across the European Union. It focuses on the institutional care received by adults with longer term mental health problems, the majority of whom have diagnoses such as schizophrenia and schizoaffective disorder. Institutions that specifically provide care for people with learning disabilities, developmental disorders or organic brain conditions including dementia are excluded in order to avoid the risk of carrying out a study with aims too broad to be able to deliver a meaningful result. We define an institution for potential inclusion in the study as a hospital or community based unit providing longer term care (i.e. not an acute admission unit) for at least seven service users in a communal setting (i.e. not individual flats/bedsits) with staff on-site, usually 24 hours a day (or if not 24 hours, the maximum number of hours provided in these kinds of units in that country). Units serviced only by outreach or "floating" support staff who are not based at the unit are excluded.

\section{Ethical approval}

East London Multi Region Ethics Committee. The ethics committees that approved the study in the other participating countries were as follows: Germany (Ethik-Kommission der Medizinischen Fakultät Carl Gustav Carus an der Technischen Universität Dresden); Czech Republic (Ethics committee of the General University Hospital, Prague); Poland (Commission of Bioethics, Wroclaw Medical University); Bulgaria (Ethics Committee of the Medical University Sofia); Portugal (Ethics Committee of the Faculdade de Ciencias Medicas, New University of Lis- aspects of the institution (such as the décor and homeliness) by the interviewer. A later stage of the study involves the assessment of service users' quality of life, autonomy, satisfaction with care and markers of Recovery. If we find good correlation between the toolkit domain ratings and service users' experiences of the institution being assessed then we can be confident that the toolkit can provide a proxy measure of the institutions' promotion of service 
bon); Spain (Comité de Ética de la Universidad de Granada); and Greece (University Mental Health Research Institutes Medical Ethics and Code of Conduct Committee, Athens).

\section{Description of the method}

\section{Phase I: The identification of "critical success factors" for recovery and internationally agreed domains for the toolkit}

vided for respondents). In the first round respondents are asked to list a maximum of ten such components or factors. In the second round participants are asked to rate each item on the overall list of components in terms of their importance in contributing to recovery on a scale of 1 (unimportant) to 5 (essential). In the third round group members are asked to adjust their ratings in light of the median group scores for each item. These responses are then analysed for consensus agreement within groups, within and across countries.

Components of care that are identified as most important to recovery from these two work streams will then be grouped into common domains agreed by the Principal Investigators at each site and an international panel with expertise in Recovery, rehabilitation, human rights legislation, mental health legislation, the rights of people with mental disabilities and care standard setting. The membership of this international expert panel is detailed in the acknowledgements for this paper.

The toolkit will include descriptive items about the institution and its service users and items to assess these agreed domains of care. The content of items and number of items per domain will reflect the nature and relative importance of each domain as evidenced from the literature review, its inclusion in care standards across countries, the Delphi exercise results and the face validity of each item. Where possible the style and format of questions will be restricted so that the "best" answer is not obvious to the interviewee and answers can be entered into an algorithm whose output summarises the nature of the unit/institution being assessed. This does not restrict scores being used for individual domains. The toolkit will be further refined in response to suggestions from the Principal Investigators in each country and the International Expert Panel. Further items will be added using the same principles as described above.

Phase 2: piloting and reliability testing the toolkit

tutional care?" (the definition of an institution is pro- 
Phase 3. Refinement of the toolkit

Phase 4: Association between toolkit assessments and service users' quality of life, autonomy and markers of recovery care using the "Your Treatment and Care" questionnaire [27] which has been extensively used in service user led audits of inpatient and community mental health care in the UK. Payments to service user participants will be made (10 Euros) to compensate them for their time and trouble in undergoing face-to-face interviews. In previous work, we have found that small payments of this kind greatly facilitate the work of researchers and preserve the dignity of participants as partners in research. Such an approach has received support from a review of payments to participants in research [28]. 


\section{Data Analysis}

\section{Phase 5. Value for money}

Analysis of Delphi exercise data

of 1-5. Overall ratings are then re-rated by participants. Items rated in the top two categories ("essential" and "very important") are collated within country and consensus ratings measured. "Strong consensus" is defined as $100 \%$ of participants being within one point of the median score, and "consensus" as $80 \%$. Consensus across partners is also analysed.

Analysis of reliability of the toolkit

multilevel modelling. Value for money will be assessed by examining associations between institution and external service costs and the toolkit domain ratings.

\section{Data management}

estimate of ICC of \pm 0.15 [31]. We shall drop items whose weighted Kappa or ICC is below 0.4. Remaining items will be subjected to a principal components factor analysis in order to obtain the smallest number that will give most information and increase the internal consistency of the factors (subscales) arising. The scree plot will be inspected to identify the point at which factors should no longer be included. Internal consistency of the core and countryspecific scales in the reduced toolkit will be assessed using Cronbach's alpha. We shall also explore the correlation of each item with the total score (item excluded), the average correlation with other items and Cronbach's alpha with that item removed.

Analysis of associations between toolkit ratings and ratings of service user quality of life, autonomy, satisfaction with care, markers of recovery and costs 
data on core toolkit scores and all users from all countries will then be undertaken with the aim of delineating the main, independent predictors in the toolkit of patients' perceived autonomy, dignity, satisfaction with care, as well as potential markers for recovery. Multilevel models will allow the analysis of service user level data (level 1 units) of perceived autonomy, dignity, satisfaction with care, and markers of recovery as the dependent variable, to investigate how institution level toolkit domain ratings (level 2 units) relate. Similar models will be used to analyse the cost data. Although the final analysis will be carried out using this method there is scarce literature informing how to choose the number of level 1 units when the number of level 2 units is fixed. Given the aim of the analysis is to explore how the core toolkit domain ratings and the service users' human rights relate to each other, it appears sufficient to assume for the purposes of a power analysis that we have 200 units (institutions). Using Dunlap and colleagues [32], it has been calculated that to be able to test for 20 predictors of a medium effect size $\left(R^{2}=0.35\right)$ with $80 \%$ power at a $5 \%$ significance level, a minimum of 170 units need to be included. These 20 predictors will be the resultant core toolkit domain ratings plus other possible institution and country level variables for which the analysis needs to be adjusted. Having multiple measures at each of these 180 units strengthens the power of the analysis since the multiple measures that will be used to estimate service users' autonomy, dignity, satisfaction with care, and markers of recovery will have less bias than a single service user measure.

A multilevel analysis of the pooled data on core toolkit scores for all users from all countries will then be undertaken. Levels likely to be important here include country, institution and service user. Using multivariable regression methods within the multilevel model will enable us to delineate the main, independent predictors in the toolkit of service users' perceived autonomy, dignity, satisfaction with care and markers of recovery.

The main output from this study will be the development of a robust toolkit for the assessment and review of the quality of care delivered to people with longer term mental health problems in hospital or community based psychiatric institutions in Europe. This is the first attempt to establish an international tool of this type. The multifaceted approach to identifying the items to be included in the toolkit (a broad systematic literature review, a qualitative Delphi exercise with key stakeholders and a review of the care standards in each participating country) and the further refinement of the toolkit though an iterative process that takes into account the results from piloting, reliability testing and the views of experts in the field, will ensure that the final product is accurate, reliable, inform- ative, useful and easy to use. The toolkit will assess aspects of care that are important across countries with very different resources and at different stages of deinstitutionalisation and that reflect the institutions' promotion of the human rights and recovery of its service users.

The study findings will be prepared for publication and for presentation at national and international conferences. Local, national and international workshops for key stakeholders will be organised to disseminate the practical implications of the toolkit. We shall also discuss the findings from the study with the World Health Organisation, the Departments of Health and care standard setting agencies in the participating countries including the practicalities of incorporating the toolkit into existing review systems for institutional care. The development of a computerised algorithm that will provide rapid toolkit scoring for comparison of institutions, as well as individualised reports for each institution based on the toolkit assessment is a further potential output from this study. Such a report could be used for the unit's own audit purposes, for local, regional or national assessments of some or all the domains of care included in the toolkit. This algorithm could be adjusted in different countries to reflect the degree to which different elements of care might be expected to be developed depending on the stage of deinstitutionalisation of the country. This computerised version of the toolkit could be completed by unit managers without the need for a face to face interview to maximise its accessibility and could complement peer review or other face to face inspections.

The development of the DEMoBinc toolkit will have obvious benefits for the individuals living in institutions providing longer term mental health care since it will provide an objective assessment of the institution's practices and systems which have a direct bearing on service users' care and treatment. The toolkit will be of direct relevance to managers and commissioners of these services since it will provide assessment of a comprehensive range of key aspects of care. It will allow the identification of problem areas that require improvement and it will provide a means to review the effect of changes in practice. It will also provide a means for the assessment of "value for money" in relation to the different domains of care. It will be able to provide information on any number of health and social care institutions and could be used for regional or national surveys of institutional care and practices. In this way it will contribute directly to the review and setting of national and, potentially international, care standards for one of the most socially excluded groups of people in Europe.

\section{Competing interests}

The authors declare that they have no competing interests. 


\section{Authors' contributions}

\section{Acknowledgements}

The study is funded by the Sixth Framework of the European Commission and the authors gratefully acknowledge this support. The authors would also like to acknowledge the contributions of the members of the International Expert Panel throughout the study and thank them for their valuable input: Mr Jerry Tew (social scientist, UK); social care - Mr Tony Ryan (independent consultant on out of area placements, UK), Mr Michael Clark (Care Services Improvement Partnership, UK); rehabilitation psychiatry and psychology - Professor Tom Craig (UK), Dr Frank Holloway (UK), Dr Jaap van Weeghel (Netherlands), Dr Joanna Meder (Poland), Professor Geoff Shepherd (UK); service user perspective - Mr Maurice Arbuthnott (UK), Ms Vanessa Pinfold (Rethink, UK); human rights law - Associate Professor Luis Fernando Barrios-Flores (University of Granada, Spain); mental health law - Professor Peter Bartlett (Nottingham University, UK); disability rights - Ms Liz Sayce (Royal Association for Disability and Rehabilitation, UK); care standards - Dr Geraldine Strathdee (Healthcare Commission, UK).

\section{References}

I. Killaspy H, Rambarran D, Bledin K: Mental health needs of clients of rehabilitation services: a survey in one Trust. Journal of Mental Health 17.

Epidemiology of schizophrenia. Psychoses of Uncertain Aetiology: Handbook of Psychiatry Volume 3

Treatment-resistant schizophrenia - the role of clozapine. Current Medical Resident Opinion I4

Neurocognitive deficits and functional outcomes in schizophrenia: are we measuring the "right stuff"? Schizophrenia Bulletin 26:

5. Green MF: What are the functional consequences of neurocognitive deficits in schizophrenia?

I53:

Cognitive deficit and the prediction of rehabilitation success in a chronic psychiatric group. 22(2):

Abnormalities of response processing in a chronic psychiatric group. A possible predic tor of failure in rehabilitation programmes?

160:

Prediction of outcome in schizophrenia: I. Relationships between predictor and outcome variables. Archives of General Psychiatry

Long-term outcome of long-stay psychiatric service users considered unsuitable to live in the community: TAPS Project 44

|8|:

virtual asylum

The NHS, the private sector and the 32:

et al. The virtual asylum: a

destructive editorial. Letter to Editor. British Medical Journal $325:$

mental illness outside the NHS: a study of out of area placements. Journal of Mental Health I3:

health out of sector placements in the North West

A census day audit of mental \& Manchester University; 2005

14. Ryan T, Hatfield B, Sharma I, Simpson V: A census day audit of mental health out of sector placements in the West Midlands Manchester: HAS CAS \& Manchester University; 2005.

15. World Health Organisation: Mental Health Atlas WHO, Geneva; 2005.
16. Liegeois A, Van Audenove C: Leiße M, Kallert TW

$$
\text { Journal of Medical Ethics }
$$

18. Goffman E:

$$
2000, \quad 450-460
$$

1961.

Doubleday Company, New York;

19. Roberts G, Wolfson P:

20. Anthony WA

$2004, \quad 37-49$.

1993, II-23.

21. Harrison G, Hopper K, Craig T,

200I, 506-5I7.

22. Mental Health Commission of New Zealand: Blueprint for menta health services in New Zealand: How things need to be.

for mental health care

The Journey to Recovery - the Government's vision

st Century. International Review of Psychiatry

the Manchester Short Assessment of Quality if Life
(MANSA).

The Resident Choice

Scale: a measure to assess opportunities for self-determination in residential settings. Journal of Intellectual Disability Research

Comparing patients' experience of mental health services in England: a five-Trust survey. International Journal of Health Quality Assurance 13(6):

What's the price of a research subject? Approaches to payment for research participation. New England Journal of Medicine 341 :

Client socio-demographic and service receipt inventory. International Outcome Measures in Mental Health

Choosing Interventions that are Cost Effective (WHO-CHOICE). [http://www.who.int/ choice/en/]. World Health Organisation

31. Streiner DL, Norman GR:

32. Dunlap $W$, Xxin X, Myers L:

Oxford University Press, Oxford; 1989 Computers

Behavior Research Methods, Instruments, \&

\section{Pre-publication history}

\section{http://www.biomedcentral.com/1471-244X/9/36/pre} pub 